\title{
Mathematical Hydraulic Models of One- Dimensional Unsteady Flow in Rivers
}

\author{
Engr. Oboshior Joel ${ }^{1}$, (MNSE), Samuel Egwunatun (MNIQS) ${ }^{2}$, Prof. (ARC.) J. E. Ahianba ${ }^{3}$ \\ ${ }^{1}$ Department of Civil Engineering ${ }^{+}$, Ambrose Alli University, Ekpoma, Edo State, Nigeria. \\ ${ }^{2}$ Delta State Polytechnic, Department of Quantity Surveying Ozoro, Delta State, Nigeria \\ ${ }^{3}$ Department of Architecture ${ }^{++}$, Ambrose Alli University, Ekpoma, Edo State, Nigeria.
}

\begin{abstract}
This flow in rivers is concerned with unsteady flow in open channel and it mathematically governed by the Saint Venant equation, using a four-point implicit finite difference scheme. For a one-dimensional applications, the relevant flow parameters are functions of time, and longitudinal positions. Considering the equations for the conservation of mass (continuity) and conservation of momentum. The mathematical method is empirical with the computer revolution, numerical methods are now effective to develop the hydraulic model. Conventional methods used to compute discharge, such as stage-discharge and stagefall discharge relationships has been inadequate. Further research is recommended to include tributaries in rivers.
\end{abstract}

Keyword-Finite difference scheme, Numerical methods, Momentum equations.

\section{INTRODUCTION}

For engineering purposes, most of the solutions of the unsteady flow equations are numerical and are determined by using digital computers. There have been a great number of different numerical techniques that could be used. Some can be discarded as being inaccurate or unstable or too time consuming. However, there is still no answer as to which is best.

Further are two fundamental divisions of methods for the solution of hyperbolic partial differential equations, describing one-dimensional unsteady flow in open channels: The hypothesis of the one dimensional flow is very often problematic when rivers with flooded plains are in question, so that the question is often raised whether the mathematical model should be one-dimensional or twodimensional, Doyle, W. H. Jr. (1983). In the case of two dimensional flow there are three independent variables: two spatial ones ( $\mathrm{x}$ ) and (y), and the time variable ( $\mathrm{t}$ ), assuming gradually varied flow conditions the flood propagation equations could be written by analogy to the tidal equations, Faye, R. E. (1984) . The system of three equations is analogous to the system derived by St. Venant for the flow in one spatial dimension. The main assumptions are the uniform distristatic vertical pressure distribution, Brakke K. (2008)

In engineering practice different methods are used for the two-dimensional modeling of flood-plains, the quasi twodimensional or "cell" model, and the mathematical twodimensional model, explicit or implicit. On the basic principles of cell model, the flood plain remains completely submerged only during the flood peak. Out of this period, the plain still forms a system of cells separated by dikes, irrigation channels, culverts, etc. all of them influencing the direction of the flow, Bakes, I. (2015). If it is possible to assume that the flood plains, as well as the river bed can be divided into a certain number of cells, then each cell communicates with the neighboring cells and the links between them correspond to an exchange of the flow, Xiaoyong Zhan (2003). The limits of the cells are defined by natural boundaries, such as are roads, dikes, natural bank levels, etc.

The cell centers must be defined so that the direction of the flow is correctly allowed for at any time during the flood. The slope of the water surface is defined for each cell (i) by the corresponding horizontal levels $\left(\mathrm{Z}_{\mathrm{i}}\right)$, assumed at the center of the cell, Fread, D. L. (2008). The area ABCD can be treated as one cell, but in this case the model would not reproduce the slope between cross-section $\mathrm{AB}$ and $\mathrm{CD}$. This means that the area $A B C D$ must be divided in two cells: $\mathrm{ABEF}$ and EFCD, with representing water level at the centers of these cells, ( $\left.T^{\prime}\right)$ and ( $\left.T^{\prime \prime}\right)$ respectively. For each cell (i) the continuity equation and discharge equation between this cell and the adjacent cells must be written. The continuity equation for a cell can be derived based on the following two fundamental hypotheses: The volume $\left(\forall_{i}\right)$ of the water stored in cell (i) is directly related to the level $\left(Z_{i}\right)$, and the dischargew between two adjacent cells (i) and $(\mathrm{k})$ is a function of the corresponding levels $\left(Z_{i}\right)$ and $\left(Z_{k}\right)$. The change in the volume of water stored in cell during time $(\Delta t)$ can be defined.

The method of characteristics deals with problems imposed by nature, but often with serious difficulties. However, this 
method has a very important role in determining the boundary values in many fixed-point finite difference methods

The general aspects of the method of characteristics was explained for St. Venant's equations written in the form of

$\frac{\partial A}{\partial 1} * \frac{\partial}{\partial x}(U . A)=-q e$

Momentum Equation

$\frac{\mathbf{1}}{\boldsymbol{g}}-\frac{\partial u}{\partial t}+\frac{u}{g} \quad \frac{\partial u}{\partial x}+\frac{1}{B} \quad \frac{\partial A}{\partial x}=S_{0}-S_{f}$

Combining the two basic equations with the two equations of the total derivatives of two dependent variables (V) and

(A),

$d V=\frac{\partial u}{\partial t} \cdot d t+\frac{\partial v}{\partial x} \cdot d x$

$d A=\frac{\partial A}{\partial t} \cdot d t+\frac{\partial A}{\partial x} \cdot d x$

and by solving the system of these four equations, the resulting characteristic equations are obtained:

$\frac{d x}{d t}=V \pm \sqrt{g \cdot \frac{A}{B}}$

$d v \pm \sqrt{\frac{g}{A \cdot B}} \cdot d A=\left[\left(S_{O}-S_{f}\right) \cdot g \pm \frac{q_{1}}{B} \cdot \sqrt{g \cdot \frac{B}{A}}\right]$

The equations (5) - (6) are four ordinary partial differential equations with the unknown values being ( $\mathrm{x} ; \mathrm{t}$; v; A). The integrals are line integrals along the characteristics. No approximations have been made.

$A\left(x, t_{0}\right)=A(x)$, and

$V\left(x, t_{0}\right)=V(x)$

The problem has to be defined for $t>$ and $\left(t_{0}\right)$ corresponds to the steady flow regime.

We assumed that in points 1, 2, 3,.., see Fig. (1B), Eqs. (7 and 8) that represent the initial conditions are known.

The objective of this paper is to fill the $(x, t)$ - plane with the net of the characteristics, so that the dependent variables can be defined at the intersections. This means, that by starting from "known" points 1, 2, 3, ..., the values of two dependent and two independent variables in points 5, 6 and 7 , can be calculated by solving the system of equations (5) and (6). Following the above described procedure, the whole $(x, t)$ - plane is filled with characteristics, as shown in Fig. (1) so that the dependent variables are defined at the intersections.

Stability is tested by asking if a particular part of the solution, perhaps an initially unimportant part, is likely to grow without limit until it destroys the calculation.

The stability is defined by the following well known equation:

$\frac{\Delta t}{\Delta \mathrm{x}} \leq \frac{1}{\left|V_{0} \pm C_{0}\right|}$

which is known as the Courant - Lewy - Fricdrichs condition, or often simply the Courant condition, where $\left(V_{0}\right)$ - is the initial value of the mean flow velocity; $\left(C_{0}\right)$ - the initial value of the small wave celerity. The boundary conditions are specified at a boundary, the number of conditions must be exactly equal to the number of characteristics originating at that boundary.

\section{METHOD}

The four possibilities for external boundaries are shown in Fig. (2)

In case a), the condition is specified at the upstream boundary and is used with the equation of the negative characteristic (R-M) to calculate the second dependent variable at point $(\mathrm{M})$.

In case b), one condition is given at the downstream boundary, and is used with the equation of positive characteristic along (L-M).

In case c), the dependent variables must be specified along the boundary so that the values at $(\mathrm{M})$ are known prior to the solution.

In case d), no boundary values are specified and the unknowns must be interpolated between (L-M) (or R- M), to obtain the dependent variables on the boundary.

In literature one can find many methods of characteristics, one of which will be presented as follows:

The basic equations (5) and (6) will be written in the following form:

- the pair of equations used for defining the positive characteristic:

$d x=(v+\sqrt{g \cdot A / B}) \cdot d t$

(10)

$d F^{+}+a^{+} \cdot d t=0$

(11)

- the air of equations used for defining the negative characteristic:

$$
d x=v-\sqrt{g \cdot \frac{A}{B}}
$$

(12)

$d F^{-}+a^{-} \cdot d t=0$

(13)

Where:

$$
F^{+}+U \pm \frac{V}{2}
$$

$$
U=\frac{\sqrt{g}}{2} \cdot \int \frac{d A}{\sqrt{A \cdot B}}
$$

$$
a^{+}=\frac{1}{2} \cdot\left[ \pm g \cdot\left(S_{f}-S_{o}\right)+\frac{q_{1}}{B} \sqrt{g \cdot \frac{B}{A}}\right]
$$

If the derivatives in the equations (14-15) are replaced by finite differences, the basic equations can be written in the following form: 
$X_{M}-X_{U}=\alpha_{U} \cdot\left(t_{M}-t_{U}\right)$

$F_{M}^{ \pm}-F_{u}^{-}+a_{u}^{+} \cdot\left(t_{M}-t_{U}\right)$

$X_{M}-X_{D}=\beta_{D} \cdot\left(t_{M}-t_{D}\right)$

$F_{M}^{-}-F_{D}^{-}+a_{D}^{-} \cdot\left(t_{M}-t_{D}\right)=0$

Where:

$\alpha_{u}=\left(V+{\sqrt{g \cdot A / B})_{U}}\right.$

$\beta_{D}=(V-\sqrt{g \cdot A / B)})_{D}$

Other symbols are explained in Fig. (3)

By using the equations (17-22), two independent unknowns in point $(M)$ are calculated first, and after that, functions $\left(F_{M}^{ \pm}\right)$and $\left(F_{M}^{-}\right)$are defined.

Two dependent variables in point $(M)$ can be calculated easily from:

$F_{M}^{ \pm}=\frac{V_{M}}{2}+U_{M}$

$F_{M}^{-}=-\frac{V_{M}}{2}+U_{M}$

$U_{M}=U(Z)$

\section{THE FINITE DIFFERENCE METHODS ON A FIXED GRID}

In contrast to the characteristic methods, most hydraulic engineers dealing with river hydraulics prefer the fixed grid methods, in order to solve the equations of motion at a finite number of grid points in the $(x, t)$ - plane. There are two basic types of fixed grid finite difference schemes: the explicit and the implicit scheme. In explicit scheme, the equations are arranged so as to find the solution for one point at a time. In an implicit scheme, a group of advance points is solved by using simultaneous equations, which include the unknowns at all points in the group.

\section{RESULT}

Finite differences using a fixed rectangular net in the $(x, t)$ plane but, it is very important to notice, that the theory of characteristics, even it is not used directly, comes into play in deciding the relative values of $(\Delta t)$ and $(\Delta x)$ which will ensure convergence. The criterion of stability is the same as for the method of characteristics, defined by Eq. 9, this being the most serious disadvantage of explicit methods. One of many existing variants of the explicit method of finite differences will be introduced, in order to explain the most important details of calculation.

The derivatives are replaced by finite differences in the following way:

$$
\left.\begin{array}{c}
\frac{\partial Z}{\partial x} \cong \frac{Z_{(p, i+1)}-Z_{(p, i+1)}}{2 . \Delta x} ; \frac{\partial Q}{\partial x} \cong \frac{Q_{(p, i+1)}-Q_{(p, i+1)}}{2 . \Delta x} \\
\frac{\partial Z}{\partial t} \cong \frac{Z_{(p, i+1)}-\bar{Z}_{(p, i+1)}}{\Delta t} ; \frac{\partial Q}{\partial x} \cong \frac{Q_{(p, i+1)}-\bar{Q}_{(p, i+1)}}{\Delta x} \\
\bar{f}_{(p, i+1)}=\frac{1}{2} \cdot\left[f_{(p, i+1)}+f_{(p, i+1)}\right]
\end{array}\right\}
$$

Where:

$$
(\bar{Z}) \text { and }(\bar{Q}) \text { the average values; }(f) \text { - any function; }(p) \text { - time row; }(i) \text { - distance row }
$$

For the explanation of basic principles of this method, the St. Venant's equations will be written in a form that corresponds more to the natural watercourses with a non-prismatic channel:

- Equation of continuity:

$$
\begin{aligned}
& \frac{\partial A}{\partial t}+\frac{\partial Q}{\partial x}=-q_{e} \\
& \frac{1}{g \cdot A} \cdot \frac{\partial Q}{\partial t}+\frac{2 V}{g \cdot A} \cdot \frac{\partial Q}{\partial x}+\left(1-F_{r}\right) \cdot \frac{\partial Z}{\partial x}= \\
& =\frac{V^{2}}{g \cdot A}\left|\frac{\partial A}{\partial x}\right|-\frac{V \cdot q_{e}}{g \cdot A}-S_{f}+D_{e}
\end{aligned}
$$

Where:

$\left(\mathrm{F}_{\mathrm{r}}\right)$ - the Froude number;

$D_{e}=\frac{V-W_{e} \cos \alpha}{g \cdot A} \cdot q_{e} ;\left(W_{e}\right)-$ velocity of the lateral flow;

$(\alpha)$ - angle of the lateral flow;

$|\partial A / \partial x|_{Z=\text { const }}$ - the term involving the influence of the non - prismatic channel.

The discretization according to Esq. (27), leads to:

- Equation of continuity:

$$
\frac{A_{(p+1, i)}-\bar{A}_{(p, i \pm 1)}}{\Delta t}+\frac{Q_{(p, i+1)}-Q_{(p, i+1)}}{2 . \Delta x}+\bar{q}_{e(p, i+1)}=0
$$

- Momentum equation: 


$$
\begin{aligned}
& \frac{Q_{(p,+1, i)}-\bar{Q}_{(p, i \pm 1)}}{\Delta t}+\frac{2 \cdot \bar{Q}_{(p, i \pm 1)}}{\bar{A}_{(p, i \pm 1)}} \cdot\left[\frac{Q_{(p, i+1)}-\bar{Q}_{(p, i-1)}}{2 \cdot \Delta x}\right]+\left[g \cdot \bar{A}_{(p, i \pm 1)}-\frac{\bar{Q}_{(p, i \pm 1)} \bar{B}_{(p, i \pm 1)}}{\bar{A}_{(p, i \pm 1) .}}\right] \cdot\left[\frac{Z_{(p, i+1)}-Z_{(p, i-1)}}{2 \cdot \Delta x}\right]= \\
& =\frac{\bar{Q}_{(p, i \pm 1)}}{\bar{A}^{2}(p, i \pm 1)} \cdot\left[\frac{A_{(p, i+1)}-A_{(p, i+1)}}{2 \cdot \Delta x}\right]_{Z=Z_{0}}+g \cdot \bar{A}_{(p, i \pm 1)} \cdot\left[-\bar{S}_{f_{(p, i \pm 1)}}+\bar{D} 1_{(p, i \pm 1)}\right]-\frac{\bar{Q}_{(p, i \pm 1)} \cdot \bar{q} 1_{(p, i \pm 1)}}{\bar{A}_{(p, i \pm 1)}}
\end{aligned}
$$

Equations (30) and (31).represent a pair of algebraic equations, that can be used for the explicit determination of dependent variables $\bar{Q}_{(p,+1, i)}$ and $Z_{(p,+1, i)}$ in the unknown point (p+1,i), see Fig.(4). The above described scheme is valid for the so called "interior" points. For the "boundary" points (points 1. And D in Fig. 4), the upstream boundary condition and the negative characteristic are used for calculating the points in cross-section (1), while the downstream boundary condition and the positive characteristic are used for the calculation of the points in the downstream cross section (D).

The equations used for calculating the points at the left boundary, in cross section $\mathrm{X}=\mathrm{X}_{1}$ are:

- the upstream boundary condition:

$$
Q_{U}=Q_{U}(t)-\text { inflow hydrograph }
$$

- the negative characteristic equation:

$$
\begin{aligned}
& \frac{Q_{(p+1,1)}-Q_{(p, 1)}}{\Delta t}+\left[V_{(p, 1)}-C_{(p, 1)}\right] \cdot \frac{Q_{(p, 2)-} Q_{(p, 1)}}{\Delta x}+B_{(p, 1)}\left[V_{(p, 1)}-C_{(p, 1)}\right] . \\
& \cdot\left\{\frac{Z_{(p+1,1)}-Z_{(p-1)}}{\Delta t}+\left[V_{(p, 1)}-C_{(p, 1)}\right] \cdot \frac{Z_{(p, 2)}-Z_{(p, 1)}}{\Delta x}\right\}=g \cdot A_{(p, 1)} \cdot\left[-S_{f(p, 1)}+D_{e(p, 1)}\right]+V_{(p, 1)}^{2} . \\
& \cdot\left[\frac{A_{(p, 1)}-A_{(p, 2)}}{\Delta x}\right]_{Z=C}+q_{e(p, 1)} \cdot C_{(p, 1)}
\end{aligned}
$$

The equations for calculating the points at the right boundary, in cross section $\mathrm{X}=\mathrm{X}_{\mathrm{D}}$, are:

- the downstream boundary condition:

$$
Q_{D}=Q_{D}(t) \text { - outflow hydrograph }
$$

- the positive characteristic equation:

$\frac{Q_{(p+1, D)}-Q_{(p, D)}}{\Delta t} \cdot\left[V_{(p, D)}-C_{(p, D)}\right] \cdot \frac{Q_{(p+1, D)}-Q_{(p, D-1)}}{\Delta x}+B_{(p, D)} \cdot\left[V_{(p, D)}+C_{(p, D)}\right] \cdot\left\{\frac{Z_{(p+1, D)}-Z_{(p, D)}}{\Delta t}+\right.$

$+\left[V_{(p, D)}+C_{(p, D)}\right] \cdot \frac{Z_{(p, D)}-Z_{(p, D-1)}}{\Delta x}=g \cdot A_{(p, D)}$.

$\cdot\left[-S_{f(p, D)}+D_{e(p, D)}\right]+V_{(p, D)}^{2} \cdot\left[\frac{A_{(p, D)}-A_{(p, D-1)}}{\Delta x}\right]_{Z=C}-q_{e(p, D)} \cdot C_{(p, D)}$

The computation by explicit scheme progresses step by s.tep; the solution is advanced to row (p+1) along the whole length of the river stretch that is analyzed.

\section{DISCUSSION}

The exact mathematical models used for commutating the unsteady flow in rivers constitute difficult mathematical problems because of the nonlinear nature of the two simultaneous partial differential equations, as has already been mentioned. Since numerical techniques are too time consuming, manual computations are practically impossible. However, as it is sometimes far too expensive to use digital computers, simplified methods called "hydraulic-hydrologic" methods are still preferred in certain cases.

The two partial differential equation (St.Venant's equations), are simplified by omitting one of the two equations (momentum equation), or by omitting certain terms in this equation. The simplified equations can be solved In several different ways, including the numerical integration by using manual successive approximation procedures. Numerous hydraulic - hydrologic methods of flood routing have been developed up to now. Descriptions of these methods is recommended for further research. By using this method, the outflow hydrograph is obtained as a result of calculation. The exactness of the quasi-steady method depends on the quality of the storage curve. This curve can be defined through hydraulic computations (computation of the water levels and of the corresponding storage of a river channel), or by balancing the differences between the inflow into a river reach and the outflow from the reach, for the flood waves registered in the past. The rate of storage can be plotted versus discharge. 




Fig. 1A: Discretization of flooded plain

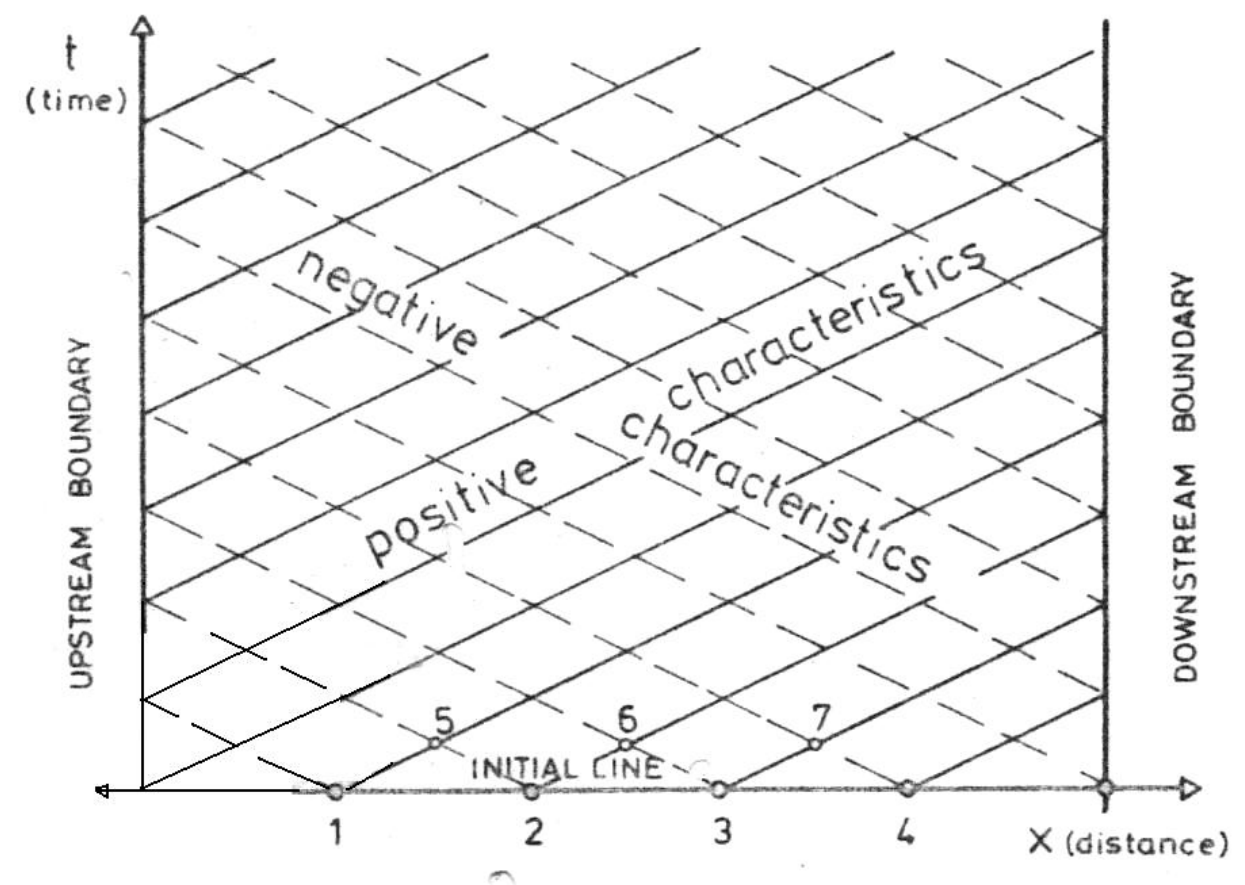

Fig. 1B: The net of characteristics 


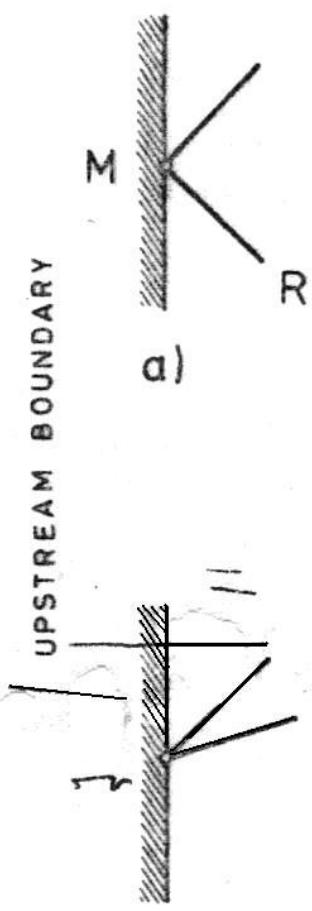

c)

\section{Subcritical flow}

\section{Superitical flow}

Fig. 2: Boundary Conditions



Fig. 3 


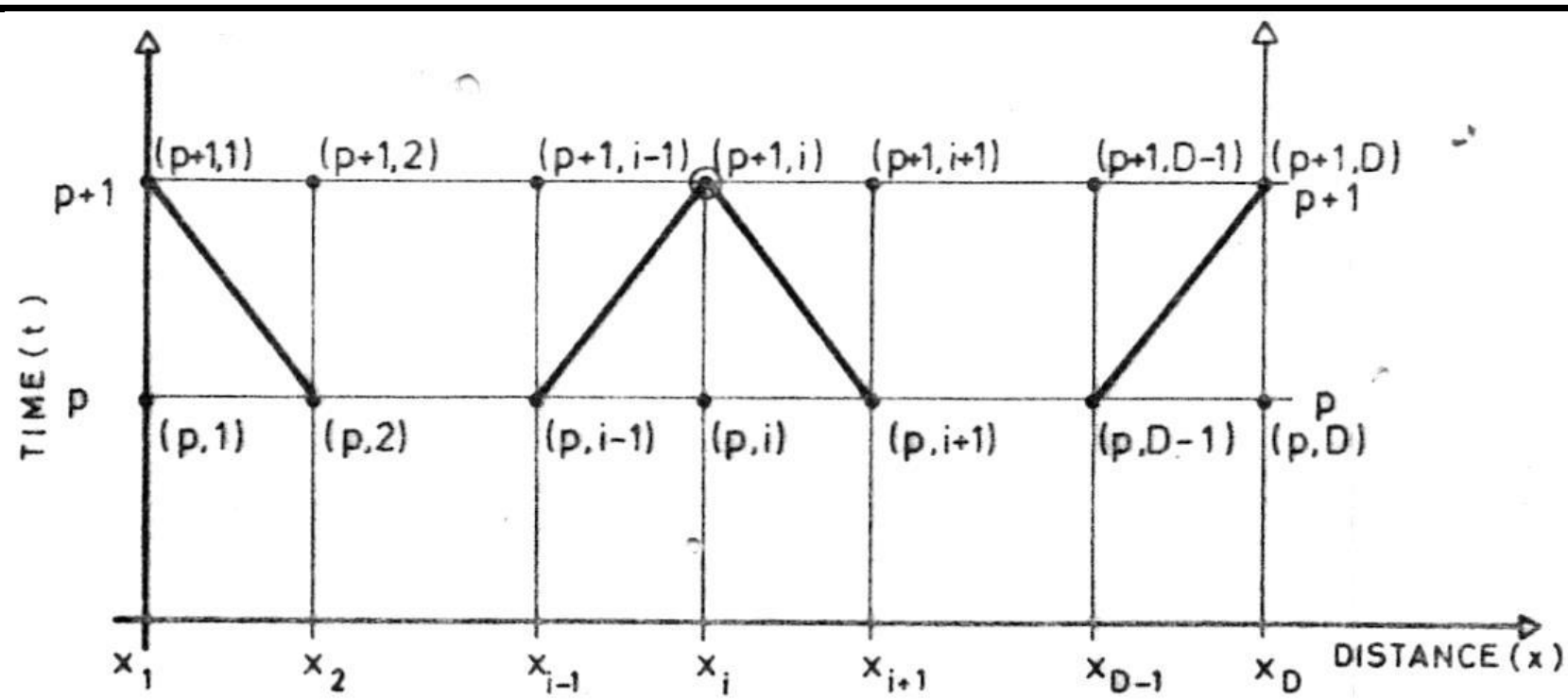

Fig. 4:

REFERENCES

[1] Bakas I. "The algebraic structure of geometric flows in two dimensions" JHEP 0510. 39.

[2] Brakke, K. The motion of a surface by its Mean Curvature, Princeton University Press, Princeton, New Jersey, 2008.

[3] Doyle W.H., Jr. Shearman, Jr O. Stiltner, G.J. and Krug, W.R., A digital model for stream flow routing by convolution methods; US Geological survey waterResources Investigations Report. 83-4160, p. 139, 1993.

[4] Faye, R.E., and Blalock, M.E., Simulation of dynamic flood flows at gaged stations in the south eastern United States; US. Geological survey water-Resources investigations Report 84-4, p. 114, 1984.

[5] Fread, D.L., Numerical pr3operties of implicit fourpoint finite difference equation of unsteady flows, HRI-45, NOAA Tech. memo NNS Hy DRO-18, Hydrologic research Laboratory, National Weather Service, Silver Spring, 2008.

[6] Xiaoyong Zhang "Simulation of unsteady flow and solute transport in a tidal river network engineering comuputations" Vol. 20 Issue. 5/6, pp. 755-768, 2003. 